\title{
Underlying Event Studies for LHC Energies
}

\author{
Gergely Gábor Barnaföldi*, András G. Agócs ${ }^{*} \dagger$ and Péter Lévai* \\ ${ }^{*}$ KFKI Research Institute for Particle and Nuclear Physics of the HAS, \\ 29-33 Konkoly-Thege M. Str. H-1121 Budapest, Hungary \\ Eötvös University, \\ 1/A Pázmány Péter Sétány, H-1117 Budapest, Hungary
}

\begin{abstract}
.
Underlying event was originally defined by the CDF collaboration decades ago. Here we improve the original definition to extend our analysis for events with multiple-jets. We introduce a definition for surrounding rings/belts and based on this definition the jet- and surrounding-belt-excluded areas will provide a good underlying event definition. We inverstigate our definition via the multiplicity in the defined geometry. In parallel, mean transverse momenta of these areas also studied in protonproton collisions at $\sqrt{s}=7 \mathrm{TeV}$ LHC energy.
\end{abstract}

Keywords: $u$ underlying event, jet physics, LHC, proton-proton collisions

PACS: 13.85.-t, 24.85.+p, 25.75.-q, 13.87.-a, 24.10.Lx

\section{INTRODUCTION}

Underlying event (UE) was originally defined by the CDF Collaboration [1] and used to investigate properties of the remaining of the events, after jets were identified and removed from there. The CDF definition of the underlying event is a simple tool in order to work, however detailed structure or information on off-jet particles cannot be obtained. On the other hand the definition is not capable to analyze more than 2-jet structures. This motivate us to develop a new definition for the underlying event.

To enhance the information content to be extracted from underlying events, we modified the above CDF's definition introducing multiple surrounding belts (SB) around the identified jets $[2,3]$. This new definition is immediately leads a more detailed analysis of the underlying event, even in case of multiple jets. On the other hand, as a specific case of our new method, one can get the originally extracted physical observables corresponding to the analysis based on the CDF-definition.

In this short contribution we present the basic properties of the two ways of defining underlying event. We recall the original CDF-based and our new definition of the underlying events, which will be compared. We used two physical quantities for our comparison: (i) the average hadron multiplicity within the defined areas and (ii) the mean transverse momenta versus multiplicity in the given regions. Quantities were investigated for both definition in parallel.

Our analysis is based on jet production and identification in proton-proton collisions at $7 \mathrm{TeV}$. We used the LHC10e14 jet-jet sample generated by PYTHIA6.2 [4] framework with cone-based UA1 [5] jet finder. 


\section{GENERALIZED DEFINITION OF THE UNDERLYING EVENT}

Any definition of underlying event should strongly depend on a jet-identification method applied in the analysis. There are various state-of-the-art development on this direction $[7,8]$, which are very promising. On the other hand there are still a problematics of these definitions - the strong process dependence. E.g. changing from proton-proton to nucleus-nucleus collisions need to re-tune the properties of the algorithms in order to find and separate jets and the baseline/background of each event [9].

The CDF-based underlying event definition corresponds to the jet identification in case of a one- (or two-) jet events. Near side jets easily define the toward and the opposite away regions of the event geometry. Our original concept was to improve the CDF-based definition on a two-folded way:

- to develope an underlying event definition which is capable to handle multijet events.

- to investigate the surrounding areas around identified jets, even without major changes of the jet-findig parameters in a case of nucleus-nucleus collision.

These requirements are led us to the definition of surrounding belts on the basis of the event-background such as 'underlying event', which completely satisfy our requirements above.

In our method, we are using jet-finding algorithms also. We define jets, than based on the physical properties of the concentrical surrounding belts and the remaining particle multiplicities, a better background or baseline can be provided. On the other hand the analysis of the surrounding area around the identified jets, can even give feedback on the goodness of the jet finding parameters.

On Fig. 1 the visual comparison of the two definitions can be seen. Left side of the figure is for the CDF-based definition, the right side displays the SB-based one. The two definitions can be summarized in a following ways, using the azimuth, $\Phi$ and (pseudo)rapidity, ( $\eta$ or) $y$ plane:

CDF-based definition of the underlying event is based on the subtraction of two areas of the whole measured acceptance: one around the identified near jet (toward region) and another to the opposite (away) direction. Both regions are $\Delta \Phi \times \Delta \eta$ slices of the measured acceptance around the near jet and to the opposite, with the full $\Delta \eta$ range and $\Delta \Phi= \pm 60^{\circ}$ in azimuth.

SB-based definition uses all identified jets of the event to subtract them from the background. Each jet can have an approximate dial-like area, around which concentric bands (or rings) can be defined. If a jet cone angle, $R=\sqrt{\Delta \Phi^{2}+\Delta \eta^{2}}$ is given, a first ' $S B_{1}$ ' and a second ' $S B_{2}$ ' surrounding belt can be defined for any jet with the thicknesses of $\delta R_{S B 1}$ and $\delta R_{S B 2}$, respectively. Generally, $\delta R_{S B i}=0.1$ with respect to the $R \approx 0.5-1$ values. It is easy to see our underlying event definition is no longer jet-number dependent.

Furthermore, increasing $\delta R_{S B i}$ values, similar (but not the same) area can be covered as in the original CDF-based definition. In this way the two model can be comparable too. 


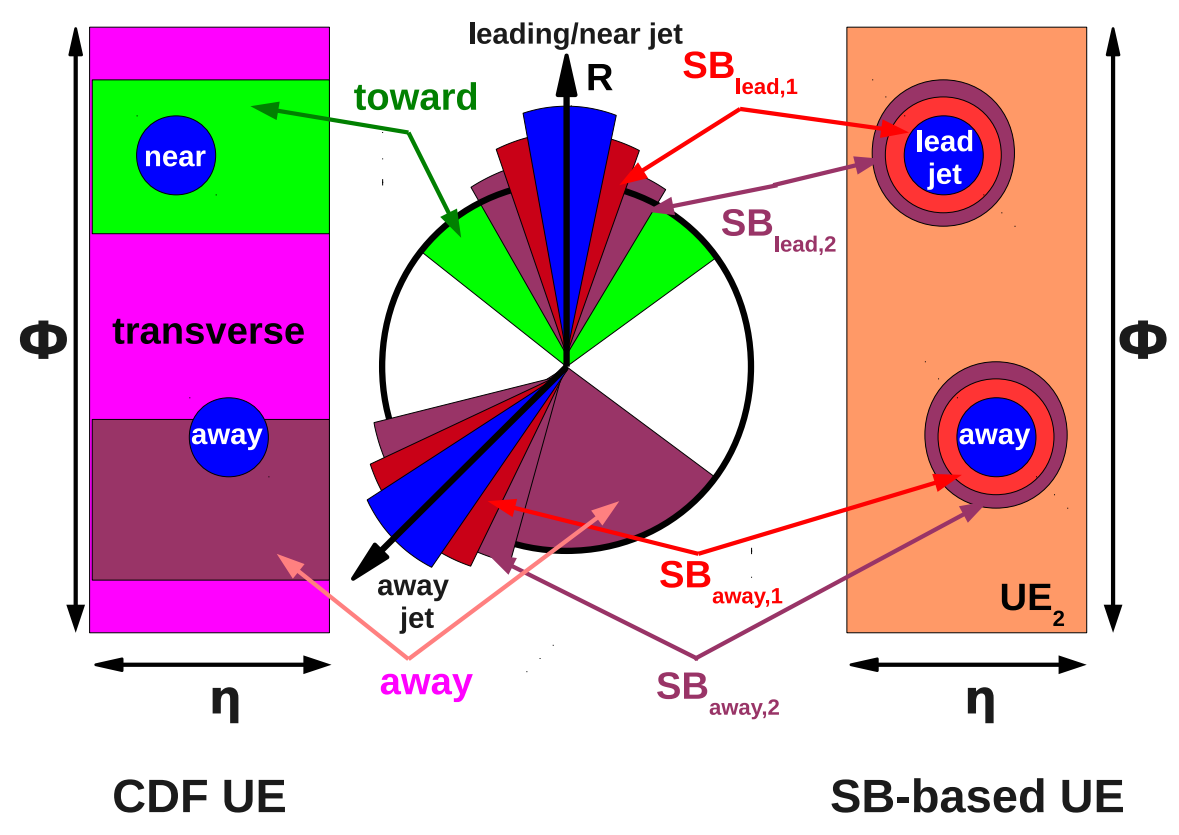

FIGURE 1. The schematic view of the underlying event (UE) defined by the CDF (left panel) and the surrounding belts (SB, right panel). Details are in the text. (Color online.)

Now we investigate the basic properties of the areas and parallel the physical quantities for the selected regions.

\section{COMPARISON OF UE DEFINITIONS}

Here we compare the details of the CDF- and surrounding belt (SB) based underlying event definitions. For our test we used PYTHIA6-simulated [4] proton-proton collisions (Perugia-0 tune [6]), namely LHC10e14 jet-jet at $7 \mathrm{TeV}$ center-of-mass energy with 150,000 events. This sample contains jets identified by UA1 method [5]. We restricted our analysis to the settings of $p_{\text {THardMin }}=10 \mathrm{GeV} / \mathrm{c}$ and $p_{\text {THardMax }}=20 \mathrm{GeV} / \mathrm{c}$.

Primarily we investigated the multiplicities of various geometrical regions of the generated events based on the full sample. After applying UA1 jet finding algorithm to identify jets, we compared the selected areas using both CDF- and SB-based definitions of the underlying events. On left panel of Fig. 2 we plotted the multiplicities, $N_{i}$ of the CDF-selected areas versus the total event multiplicity. Here $N_{i}$ refers for followings: the multiplicities of the identified 'leading/near jet' (blue squares), the jet-excluded 'toward' area (green dots), the 'away' side area to the opposite direction (purple dots), and the CDF-defined underlying is event, 'transverse' (pink dots). The right side of the Fig. 2 stands for the SB-based underlying event definition with more areas: the multiplicities of the identified leading jet blue squares), the away side jet (blue dots), multiplicity for the surrounding belts, $S B_{\text {lead }, 1}, S B_{\text {lead }, 2}, S B_{\text {away, },}$, and $S B_{\text {away,2 }}$ are open red squares, open purple triangles, open red circles, open purple diamonds respectively. Finally orange 

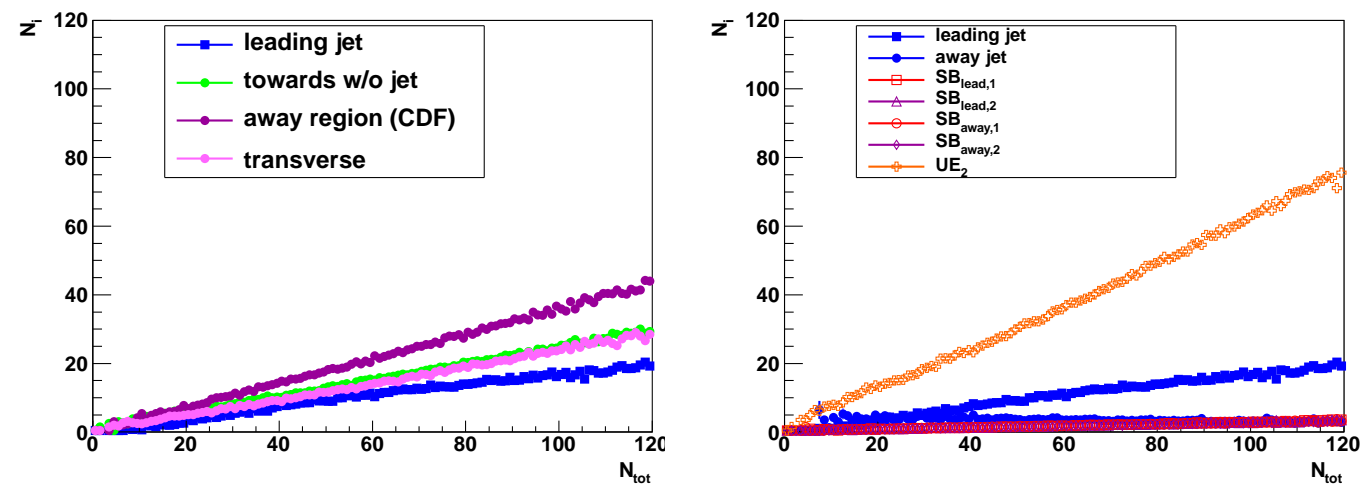

FIGURE 2. The multiplicity, $N_{i}$ for the selected areas as the multiplicity of the total event, $N_{\text {tot }}$. Underlying event regions are defined on the left panel for the CDF-based and on the right panel for the surrounding belt based definitions. More details are in the text. (Color online.)

crosses denote multiplicity for the newly defined underlying event $U E_{2}$ outside all jets. (Note, all color in accordance with the areas of Fig 1 above.)

Fig. 2 shows multiplicity in almost all regions: $N_{i}$ increases almost linearly with the total multiplicity, in the $N_{t o t}<120$ region of the event for both cases. In case of the CDFbased definition, the away region gives the biggest contribution, and the jet belongs to the smallest one. The transverse (underlying event) area lies between the two extremal contribution. Moreover, it is interesting to see, after excluding the jet from the toward region, the remaining area has almost the same multiplicity as the underlying event. This shows the goodness of the jet finding algorithms and the "safety" of the CDFbased underlying event definition (e.g. 1/3 of the whole acceptance far from any jetcontaminated areas).

The multiplicity relations of the SB-based definition differs from the CDF-based. The near jet has the same contribution, away side jet and the $S B_{i}$ s have small fraction from the $N_{\text {tot }}$ - due to the small areas. On the other hand, the newly defined underlying event, $U E_{2}$ dominates the event multiplicity since it has almost the whole acceptance.

In general the multiplicity fraction of the defined areas are almost proportional to the geometrical surface, only the jet-content part violates this dependence, as Fig. 2 displays. Thus, the SB-based $U E_{2}$ has larger multiplicity comparing to the CDF-based one, which might gives better statistics for an underlying event analysis.

Secondly, the mean transverse momentum $\left\langle p_{T}\right\rangle$ of the selected areas is investigated including especially the underlying event. We plotted the $\left\langle p_{T}\right\rangle$ vs. the multiplicity of the total event, $N_{\text {tot }}$ and the $\left\langle p_{T}\right\rangle$ vs. multiplicity of the CDF-based and SB-based underlying events.

On Fig. 3 we display the $\left\langle p_{T}\right\rangle$ vs. the multiplicity of the total event, $N_{\text {tot }}$ for both CDF-based (left panel) and SB-based (right panel) underlying event. We use the same color and mark encoding for the selected regions of the event as on Fig. 2 above.

We found the mean- $p_{T}$ distributions of the regions are similar in proton-proton collisions. The identified leading jet has the highest values $\left\langle p_{T}\right\rangle_{\text {leading }}$ jet $\sim 8-9 \mathrm{GeV} / \mathrm{c}$, which are decreasing to $\left\langle p_{T}\right\rangle_{\text {leading jet }} \sim 5-6 \mathrm{GeV} / \mathrm{c}$ as going to larger $N_{\text {tot }}$, for both 

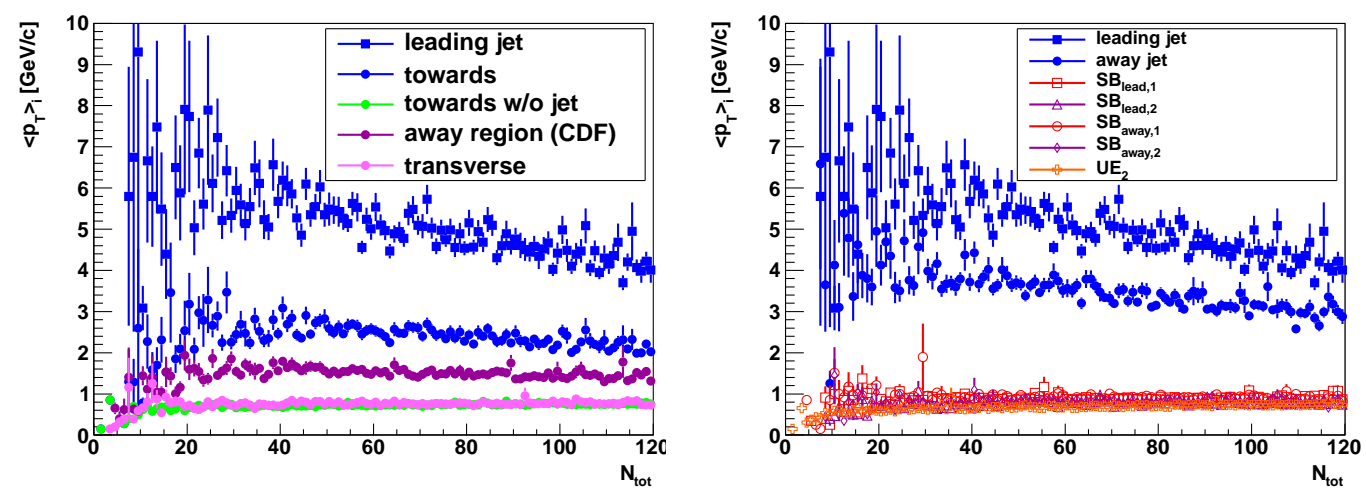

FIGURE 3. The average transverse momenta versus the total multiplicity of the events, $N_{\text {tot }}$. Regions defined on the left panel are for the CDF-based and on the right panel are for the SB-based definitions. More details are in the text. (Color online.)

definition's cases. The mean- $p_{T}$ for both underlying event cases are the same with the constant value $\left\langle p_{T}\right\rangle_{U E i} \sim 0.5 \mathrm{GeV} / \mathrm{c}$. For the CDF-based definition this is similar to the jet-excluded toward area also, and for the SB-based definition surrounding belts, $S B_{i}$ have also similar, but a slightly higher $\left\langle p_{T}\right\rangle_{S B i} \sim 1.0 \mathrm{GeV} / \mathrm{c}$. Differences between the left and the right panels are originating from the handling of the near and away side jet. CDF-based definition contains the jet to the near (leading) direction, and fully or partially to the opposite away region: $\left\langle p_{T}\right\rangle_{\text {towards }} \sim 2-3 \mathrm{GeV} / \mathrm{c}$ and $\left\langle p_{T}\right\rangle_{\text {away }} \sim 1.5-2$ $\mathrm{GeV} / \mathrm{c}$. SB-based case since away side jets were also identified, $\left\langle p_{T}\right\rangle_{\text {away }}$ jet $\sim 2-3$ $\mathrm{GeV} / \mathrm{c}$. We can state generally, both underlying-event definition give the same result and they are differ only in the separation (or inclusion) of leading or away side jet to the given areas.
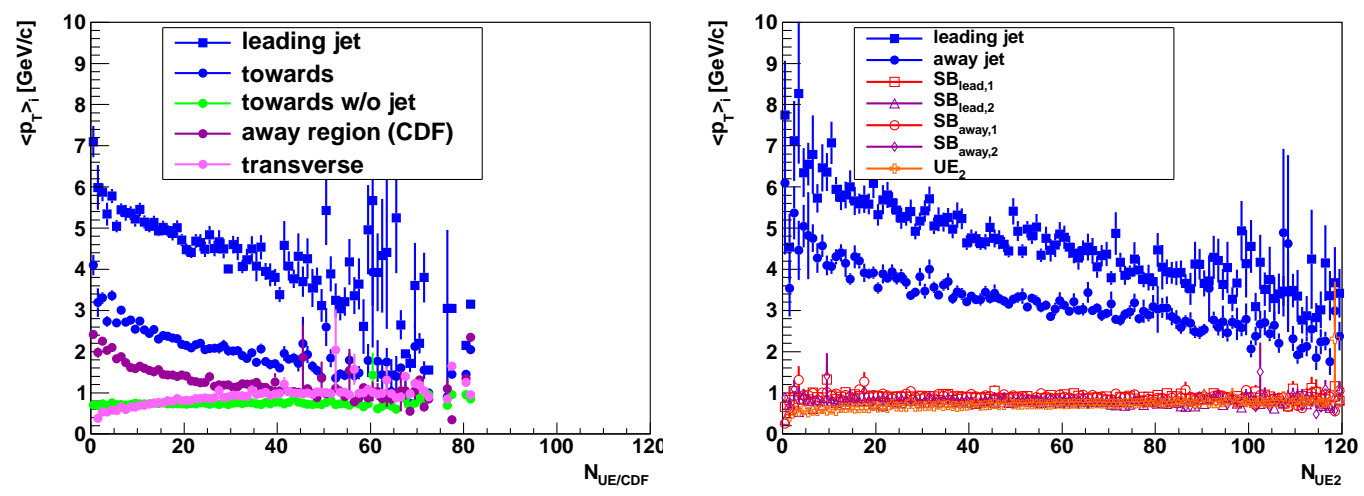

FIGURE 4. The average transverse momenta versus the multiplicity of the underlying evens. Regions are defined on the left panel for the CDF UE and on the right panel for the surrounding belt based UE definitions. More details are in the text. (Color online.)

Finally on Fig. 4 we compared mean- $p_{T}$ values vs. to the self-definition-given underlying events: CDF-based UE, $N_{U E / C D F}$ on left panel and SB-based $N_{U E 2}$ on right panel. 
(Colors and marks are the same as above figures.) Here, the comparison shows slight difference between the panels. A stronger decrease in the highest $\left\langle p_{T}\right\rangle$-content regions present compared to Fig. 3. Furthermore, changing from $N_{t o t}$ to $N_{U E / C D F}$ and $N_{U E 2}$ the separation of the curves are more clear in both cases, especially at the largest $\left\langle p_{T}\right\rangle$ values. In parallel the mean- $p_{T}$ values for the underlying event are almost the same for the average multiplicity events and slightly higher for the rare ones.

\section{CONCLUSIONS}

We studied our new underlying event definition in $\sqrt{s}=7 \mathrm{TeV}$ proton-proton collisions with 150,000 events. We investigated and compared the multiplicities and the mean- $p_{T}$ vs. multiplicities for the CDF-based and our SB-based definition.

We found the multiplicity fraction of the defined regions are almost proportional to the geometrical surface, only the jet-content part differs, due to the separation (or inclusion) of the leading or away side jets. The SB-based underlying event, $U E_{2}$ found to have larger multiplicity comparing to the CDF-based, $N_{U E / C D F}$ one, which might gives better statistics for the underlying event analysis.

The mean- $p_{T}$ vs. $N_{t o t}$ analysis led us to compare both definition on the same level. We got the same dependence of the underlying event for both, CDF- and SB-based cases. On the other hand, the above mentioned jet and near/away-area handling leads to differences.

Finally, we compared our definitions by the mean- $p_{T}$ vs. the self-defined underlying event multiplicities, namely $N_{U E / C D F}$ and $N_{U E 2}$. Our results have shown both definition is reliable, and - due to the generalized definition of the surrounding belt based underlying event - multiple jets and detailed analysis of the surrounding areas can be performed in the future.

\section{ACKNOWLEDGMENTS}

This work was supported by Hungarian OTKA NK77816, PD73596 and Eötvös University. Authors (GGB \& PL) are appreciate the local support by the UNAM, Mexico and GGB thanks for the János Bolyai Research Scholarship of the HAS.

\section{REFERENCES}

1. A. A. Affolder et al. [CDF Collaboration], Phys. Rev. D65, 092002 (2002).

2. P. Lévai and A. G. Agócs, PoS EPS-HEP2009, 472 (2009).

3. A. G. Agócs, G. G. Barnaföldi, and P. Lévai, arXiv:1011.5363 [hep-ph], accepted for the Proc. of Hot Quarks 2010 Workshop.

4. T. Sjostrand, S. Mrenna, and P. Z. Skands, JHEP 0605, 026 (2006).

5. G. Arnison et al. [UA1 Collaboration], CERN-EP/83-118, Phys.Lett. B132, 214 (1983).

6. P. Z. Skands, MCNET-10-08, CERN-PH-TH-2010-113, Phys.Rev. D82, 074018 (2010).

7. G. P. Salam, Eur. Phys. J. C67, 637-686 (2010).

8. M. Cacciari, G. P. Salam, S. Sapeta, JHEP 1004, 065 (2010).

9. S. Salur, Nucl. Phys. A830, 139 (2009). 